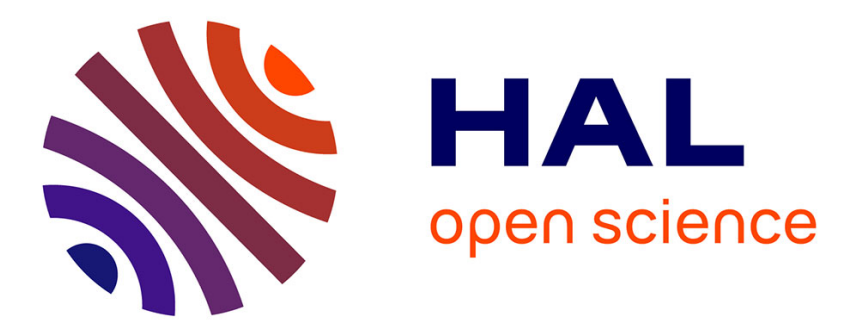

\title{
Experimental and theoretical characterization of mixed mode brittle failure from square holes
}

\author{
Aurélien Doitrand, Pietro Cornetti, Alberto Sapora, Rafael Estevez
}

\section{To cite this version:}

Aurélien Doitrand, Pietro Cornetti, Alberto Sapora, Rafael Estevez. Experimental and theoretical characterization of mixed mode brittle failure from square holes. International Journal of Fracture, 2021, 228 (1), pp.33-43. 10.1007/s10704-020-00512-9 . hal-03336440

\author{
HAL Id: hal-03336440 \\ https://hal.science/hal-03336440
}

Submitted on 7 Sep 2021

HAL is a multi-disciplinary open access archive for the deposit and dissemination of scientific research documents, whether they are published or not. The documents may come from teaching and research institutions in France or abroad, or from public or private research centers.
L'archive ouverte pluridisciplinaire HAL, est destinée au dépôt et à la diffusion de documents scientifiques de niveau recherche, publiés ou non, émanant des établissements d'enseignement et de recherche français ou étrangers, des laboratoires publics ou privés. 


\title{
Experimental and theoretical characterization of mixed mode brittle failure from square holes
}

\author{
Aurélien Doitrand · Pietro Cornetti • Alberto Sapora • \\ Rafael Estevez
}

Received: date / Accepted: date

\begin{abstract}
Mixed mode crack initiation in PMMA specimens containing square holes is investigated experimentally by uniaxial tensile tests. The crack deflection with respect to the V-notch bisector and the failure load both increase for decreasing hole sizes, to which correspond higher mode mixities. In order to catch this behavior from a theoretical point of view, Finite Fracture Mechanics (FFM) approach is employed numerically through a full finite element analysis. The FFM implementation requires only two material parameters, namely the strength and the fracture toughness. They are identified through the comparison between failure load predictions and experimental measurements on circular holes with different diameters. The resulting FFM blind predictions on square holes are in good agreement with recorded data. Finally, FFM is implemented through an asymptotic matching expansion procedure and an excellent matching with the previous simulations is found.
\end{abstract}

Keywords Square hole $\cdot$ PMMA · uniaxial test $\cdot$ Mixed mode failure $\cdot$ Finite Fracture Mechanics $\cdot$ circular hole

\footnotetext{
Aurélien Doitrand, corresponding author

Université Grenoble-Alpes - CNRS UMR 5266, SIMaP, F-38000 Grenoble, France

Present adress:Université Lyon, INSA-Lyon, MATEIS UMR CNRS 5510, F-69621 Villeurbanne Cedex, France

Tel.: +33472438803

E-mail: aurelien.doitrand@insa-lyon.fr

Pietro Cornetti

Department of Structural, Geotechnical and Building Engineering, Politecnico di Torino, 10129 Turin, Italy

Alberto Sapora

Department of Structural, Geotechnical and Building Engineering, Politecnico di Torino, 10129 Turin, Italy

Rafael Estevez

Université Grenoble-Alpes - CNRS UMR 5266, SIMaP, F-38000 Grenoble, France
} 


\section{Introduction}

Mixed mode failure in brittle materials can be assessed employing classical tools from linear elastic fracture mechanics (LEFM) only in presence of sufficiently large cracks, when the stress intensity factors (SIFs) govern the singular stress field in the neighborhood of the crack tip. In the case of short cracks, notches or holes, it is necessary to implement more powerful theories, such as Finite Fracture Mechanics (FFM) [13, 18, which is based on the assumption of a finite crack increment, at least at the first step. In this framework, Leguillon firstly introduced the Coupled Criterion (CC) [16], which allows the prediction of crack initiation by combining both stress and energy requirements. Following his idea, Yosibash et al. [19,20,23] and, later on, Sapora et al. [5,21] modeled mixed mode crack initiation occurring from $\mathrm{V}$-notches.

The CC can be employed through different approaches. The Finite Element (FE) implementations of the CC have been recently recalled and gathered in a tool for Abaqus $^{T M}$ [10]. The first approach, initially proposed by Leguillon [16], relies on a matched asymptotic (MA) expansion procedure [15. It consists in computing the stress and the energy conditions in a domain surrounding the initiation crack location without considering the whole geometry. The boundary conditions imposed to this domain are the asymptotic displacements or stress fields corresponding to the singular point or the stress raiser. They depend on the generalized stress intensity factors (GSIFs) related to opening and in-plane shear modes: the procedure reveals to be convenient to compute the stress and the energy criteria for a given mode mixity. This approach was traditionally and successfully employed to predict mixed mode failure initiation [8,20,23, for instance at V-notches [19,21,23. Furthermore, Felger et al. [1] predicted mixed mode crack initiation at bi-material junctions under four point bending based on the MA approach, taking into account two possible failure mechanisms, namely the bending failure or the notch induced failure. An asymptotic approach was also employed by Cornetti et al. [5] to predict crack initiation of specimens containing square holes under either uniaxial or bi-axial loading, which leads to mixed mode crack initiation from the hole corners, the mode mixity depending on the notch size. Failure stress and crack deflection were computed semi-analytically for several self-similar specimens, highlighting the ability of the CC to catch size effects.

The second CC approach consists in performing full finite element (FFE) simulations [3, 6, 7, 9, 17, This method requires to model the whole structure under investigation, and it reveals to be necessary when the defect/notch size or the crack length are not small enough with respect to the other geometrical dimensions, i.e. when the solution is not governed by the GSIFs. It is important to remind that both MA and FFE approaches 
require not only the failure load and the initiation crack length to be determined, but also the crack initiation angle. This is usually achieved by computing the failure load for several angles and by determining the one minimizing the failure stress.

Mixed mode crack initiation from notches was studied experimentally by several authors. Seweryn and Lukaszewicz 22. performed Arcan tests on double V-notch specimens with several opening angles and thus different mode mixities, and computed critical loads and crack orientations using several fracture criteria. Three and four point bending tests on V-notch specimens were performed respectively by Yosibash et al. [23] and Priel et al. [19]. In both cases, the mode mixity was obtained by varying the position of the loading point and of the supports. Another way to generate mixed mode loading under three point bending of V-notch specimens was employed by Gómez et al. [12] who manufactured specimens with a slanted V-notch. Ayatollahi and Aliha [1] designed the so-called diagonally loaded square plate test that allows providing pure mode I or mode II loading conditions as well as any mixed mode conditions in between, showing a good agreement with classical mixed mode fracture criteria. Brazilian Disk tests on specimens with a central square hole were performed by Ayatollahi et al. [2]. Using stress-based criteria, they showed the necessity of employing an additional parameter (a critical distance in their work) to the material strength in order to correctly predict the crack initiation location. The proposed approach did not allow the initiation crack extension to be predicted, and no information was given about the experimental identification of the critical distance.

The present work deals with the experimental and theoretical characterization of size effects in structures containing square holes. Experimental tests on PMMA samples are described in Section 2 , whilst the coupled criterion theory and its FFE implementation are introduced in Section 3 Finally, in Section 4 , the material fracture parameters are identified, and numerical predictions on the failure load and crack deflection -as a function of the hole size- are successfully compared with experimental data.

\section{Experimental campaign}

The material under investigation is a commercial extruded polymethyl methacrylate (PMMA) supplied by NUDEC. The related Young's modulus $(E=2.7 \pm 0.1 \mathrm{GPa})$ and Poisson's ratio $(\nu=0.39 \pm 0.005)$ were measured by testing plain specimens and using both extensometers and digital image correlation techniques.

Two types of notches were laser cut on rectangular samples having thickness $t=5.76 \pm 0.05 \mathrm{~mm}$ and length $L=150 \pm 0.2 \mathrm{~mm}$. The first one refers to circular holes with diameter $d$. This kind of geometry was considered in 

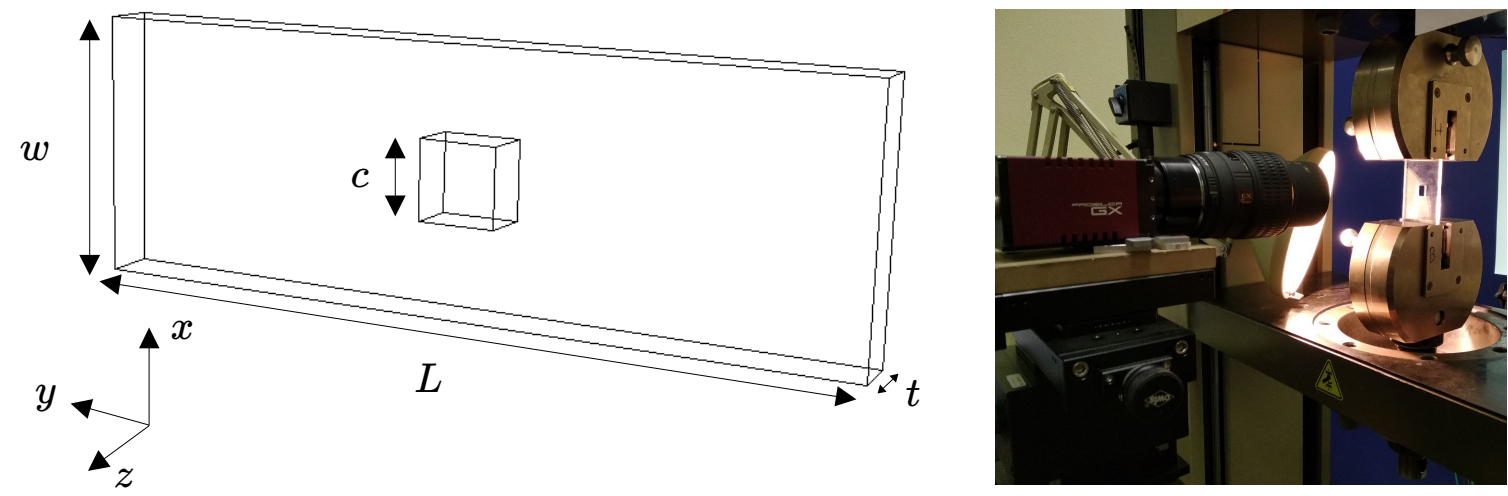

Fig. 1: Geometry of square hole specimens and experimental setup.

order to identify the material strength $\sigma_{c}$ and the fracture toughness $G_{c}$, necessary for the FFM implementation. The second one is related to square holes with side $c$ parallel to the specimen side $w$ (cf. Fig. 1). Such a configuration was chosen because it results in mixed mode crack initiation, the mode mixity $\psi$ depending on the hole size as described in [5]. Different geometrical dimensions were considered for both notches, and three specimens for each configuration were tested using a $20 \mathrm{kN}$ Zwick machine ( $c f$. experimental set-up, Fig. 1). The force and displacement were acquired every $0.1 \mathrm{~s}$. . The prescribed displacement rate was $0.5 \mathrm{~mm} / \mathrm{min}$, which was small enough to ensure an overall quasi-static loading state. The tests were optically recorded at $1 \mathrm{~Hz}$ frequency with 1s exposure time using a 29M Pixels Allied Vision Prosilica GX camera. Further details are given below.

\subsection{Uniaxial tests on circular hole specimens}

Five different circular hole geometries were machined and tested, with a width to diameter ratio $w / d$ varying between 7.3 (for the smallest hole) and 9.3 (for the largest hole). The specimen dimensions are reported in Table 1. Under tensile loading, mode I cracking occurs in circular hole specimens: the crack initiating from the hole that acts as a stress raiser and the specimen breaks into two parts. A photograph of one circular hole specimen for each configuration is shown before (Fig. 3) and after (Fig. 4) failure.

Load-displacement curves are shown in Fig. 2 and the corresponding failure loads are reported in Table 1 . They reveal decreasing failure stresses (where the stress is defined as the ratio between the failure load and the

\begin{tabular}{|c|c|c|c|c|c|}
\hline Circular hole diameter $d(\mathrm{~mm})$ & $0.66 \pm 0.12$ & $1.22 \pm 0.27$ & $2.31 \pm 0.22$ & $3.37 \pm 0.26$ & $4.28 \pm 0.28$ \\
\hline Specimen width $w( \pm 0.2 \mathrm{~mm})$ & 4.80 & 9.80 & 19.80 & 29.80 & 39.80 \\
\hline Failure Load $(\mathrm{N})$ & $585 \pm 41$ & $1050 \pm 101$ & $1633 \pm 46$ & $2442 \pm 245$ & $3298 \pm 390$ \\
\hline
\end{tabular}

Table 1: Experimental results on circular holes: geometrical dimensions and recorded failure loads. 


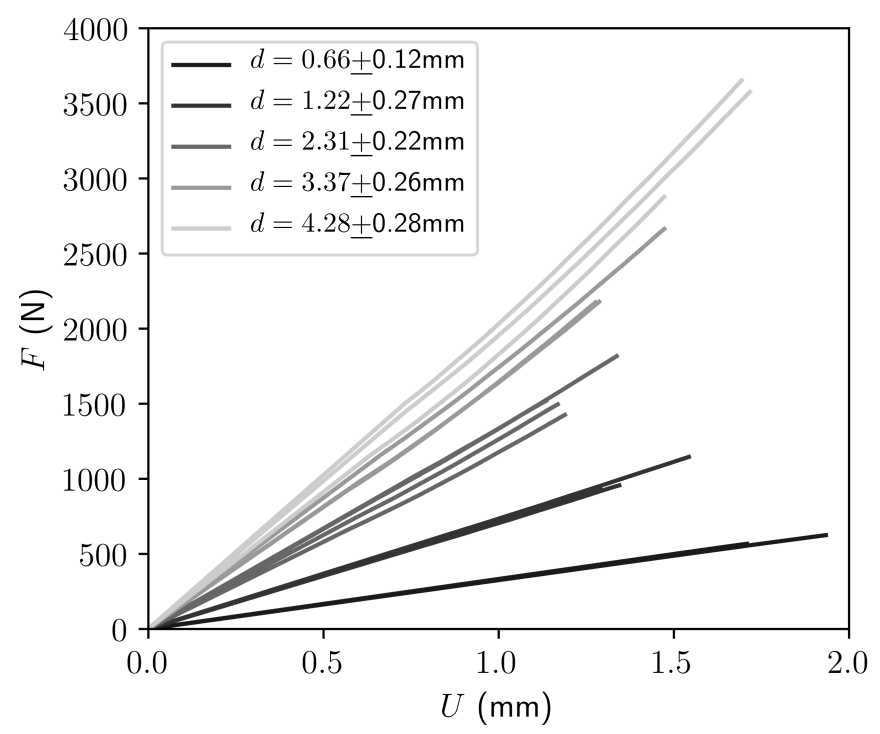

Fig. 2: Load-displacement curves corresponding to the specimens containing circular holes.

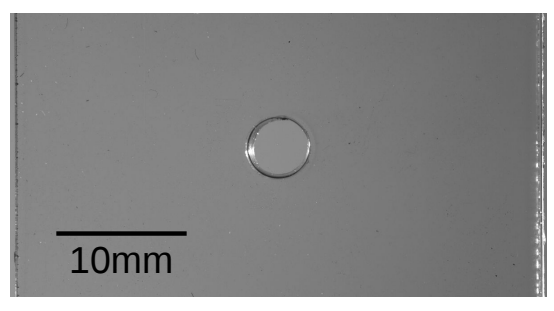

(a)

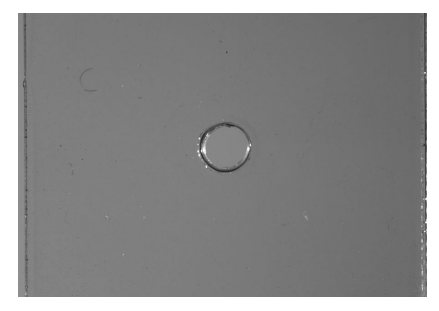

(b)

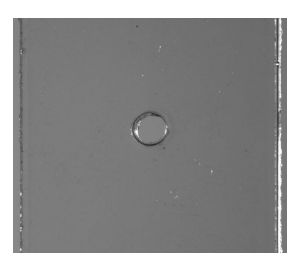

(c)

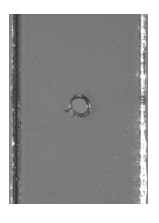

(d)

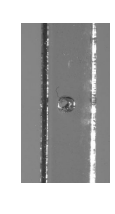

(e)

Fig. 3: Specimens with circular hole sizes varying between (a) $4.28 \mathrm{~mm}$ and (e) $0.66 \mathrm{~mm}$ before failure.

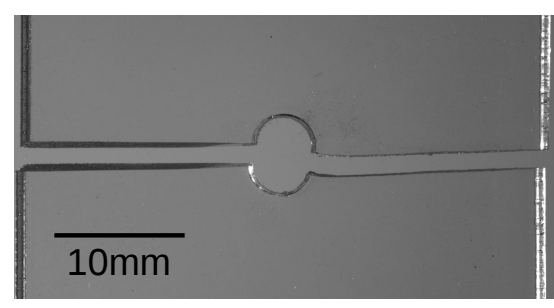

(a)

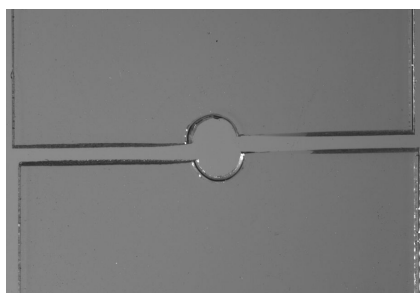

(b)

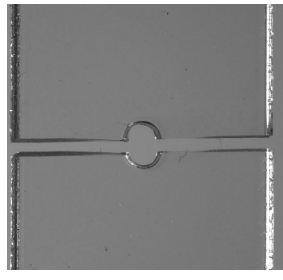

(c)

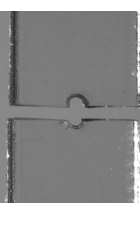

(d)

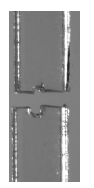

(e)

Fig. 4: Specimens with circular hole sizes varying between (a) $4.28 \mathrm{~mm}$ and (e) $0.66 \mathrm{~mm}$ after failure.

specimen section $w \times t$ ) for increasing hole diameters. Indeed, for sufficiently large hole size, the failure stress tends towards the asymptotic value $\sigma_{c} / 3$. As anticipated before, experimental data obtained from circular hole specimens will be exploited in Section 4.1 to determine the material strength $\sigma_{c}$ and fracture toughness $G_{c}$. These values will be used, in turn, for blind predictions on crack initiation from square holes. 


\subsection{Uniaxial tests on square hole specimens}

A total of 10 square hole configurations were considered. Three specimens were tested for each configuration were tested by varying the hole side from $1.36+0.27 \mathrm{~mm}$ to $15.3 \pm 0.28 \mathrm{~mm}$. The geometrical dimensions are reported in Table 2, Under tensile loading, mixed mode cracking occurs in square hole specimens, the cracks initiating from the square hole $\mathrm{V}$-notches that act as stress raisers. A photograph of one square hole specimen for each configuration is shown before (Fig 5) and after (Fig. 6) fracture. Note that the influence of the notch radius will be disregarded in the present study, since relatively small compared to the hole size. The failure loads for the different samples and hole sizes are reported in Table 2, Two propagation cracks were observed for all the tested configurations. These cracks initiate and instantaneously propagate so that they break the specimen

\begin{tabular}{|c|c|c|c|c|c|}
\hline Square hole side $c(\mathrm{~mm})$ & $1.36 \pm 0.27$ & $1.75 \pm 0.3$ & $2.38 \pm 0.2$ & $2.8 \pm 0.2$ & $3.53 \pm 0.14$ \\
\hline Specimen width $w( \pm 0.2 \mathrm{~mm})$ & 9.80 & 14.80 & 19.80 & 24.80 & 29.80 \\
\hline Failure load $(\mathrm{N})$ & $1223 \pm 92$ & $1401 \pm 121$ & $1765 \pm 167$ & $2053 \pm 145$ & $2214 \pm 104$ \\
\hline Crack deflection $($ deg. $)$ & $43 \pm 5.3$ & $38.4 \pm 14.1$ & $42.9 \pm 3.0$ & $37.2 \pm 4.3$ & $36.1 \pm 1.4$ \\
\hline \hline Square hole side $c(\mathrm{~mm})$ & $5.04 \pm 0.08$ & $6.36 \pm 0.41$ & $9.39 \pm 0.27$ & $12.34 \pm 0.18$ & $15.34 \pm 0.28$ \\
\hline Specimen width $w( \pm 0.2 \mathrm{~mm})$ & 29.80 & 29.80 & 49.80 & 49.80 & 49.80 \\
\hline Failure load $(\mathrm{N})$ & $1863 \pm 121$ & $1702 \pm 84$ & $2357 \pm 146$ & $2206 \pm 220$ & $2093 \pm 204$ \\
\hline Crack deflection (deg.) & $33.4 \pm 7.4$ & $31.1 \pm 9.3$ & $31.4 \pm 11$ & $24.0 \pm 10.7$ & $22.7 \pm 5.09$ \\
\hline
\end{tabular}

Table 2: Experimental results on circular hole specimens: failure loads and critical deflection angles.

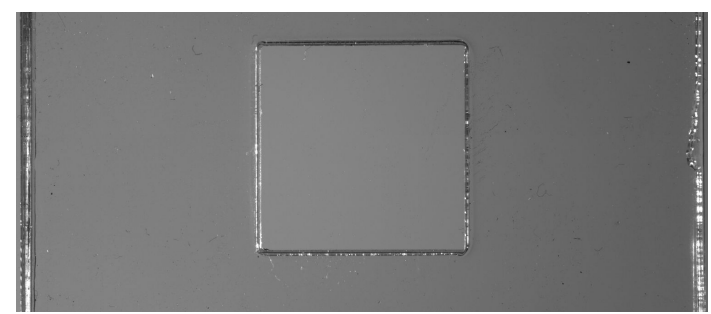

$10 \mathrm{~mm}$

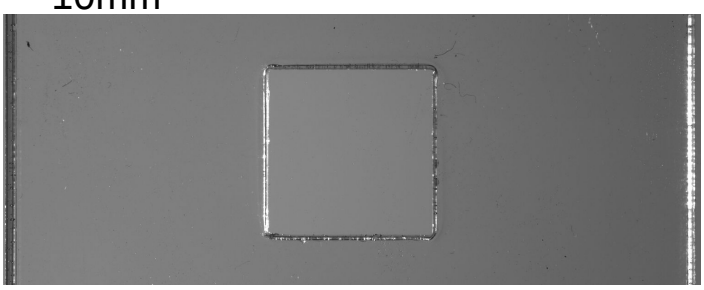

(b)

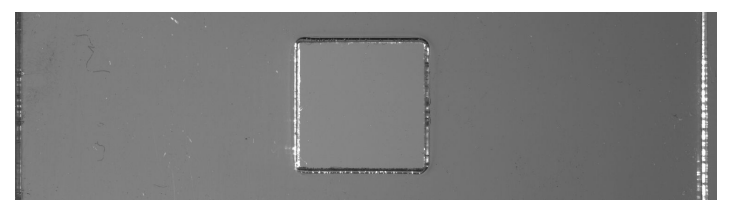

(c)

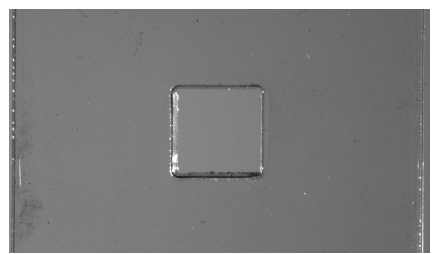

(d)

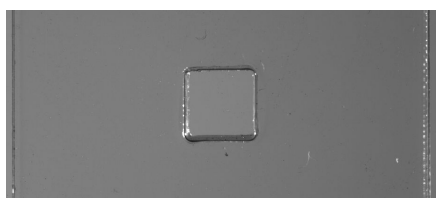

(e)

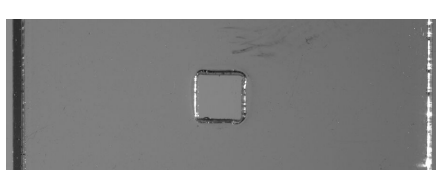

(f)

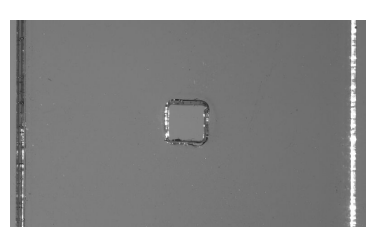

(g)

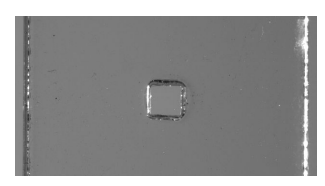

(h)

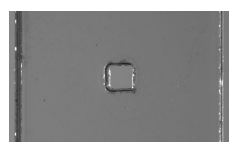

(i)

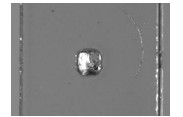

(j)

Fig. 5: Specimens with a square hole size varying between (a) $15.34 \mathrm{~mm}$ and (i) $1.36 \mathrm{~mm}$ before failure. 


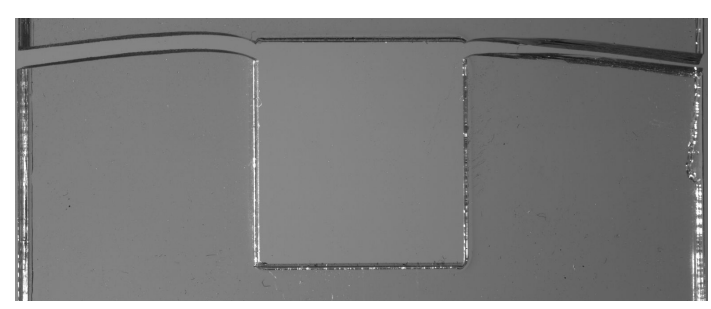

$10 \mathrm{~mm}$

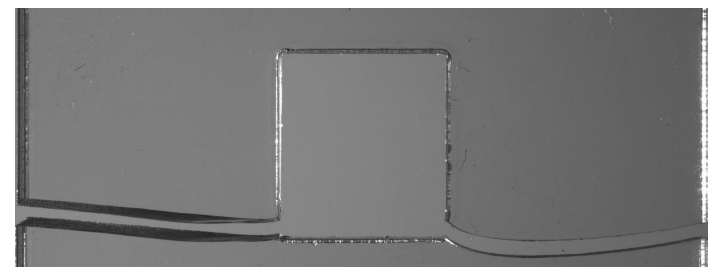

(b)

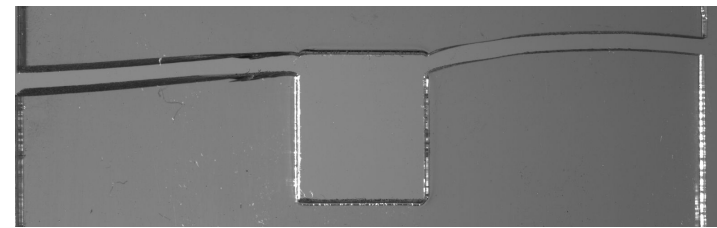

(c)

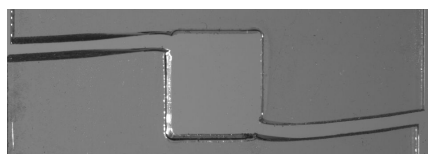

(d)

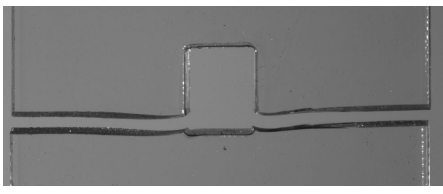

(e)

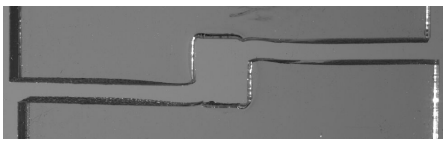

(f)

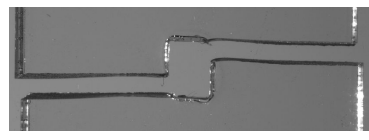

(g)

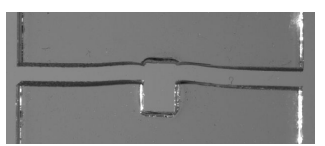

(h)

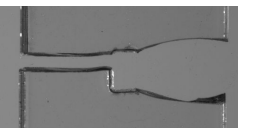

(i)

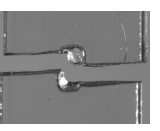

(i)

Fig. 6: Specimens with a square hole size varying between (a) $15.34 \mathrm{~mm}$ and (i) $1.36 \mathrm{~mm}$ after failure.

into two parts. They are located either both at the top or bottom corners (Fig 6a-c,e,h) or at opposite corners (Fig 6 $\mathrm{d}, \mathrm{f}, \mathrm{g}, \mathrm{j})$. They correspond to a decreasing failure stress with increasing hole size. Similarly to circular hole specimens, the specimen behaves like if there was no defect when the hole size tends towards zero, so that the failure stress tends towards the material strength. The crack deflections with respect to the square hole V-notch bisectors for the different hole sizes are reported in Table 2. These deflections were measured through microscope observations on the square hole corners after failure in an area around $0.5 \mathrm{~mm}$ from the V-notch edge for which the crack path is straight(Fig. 7).

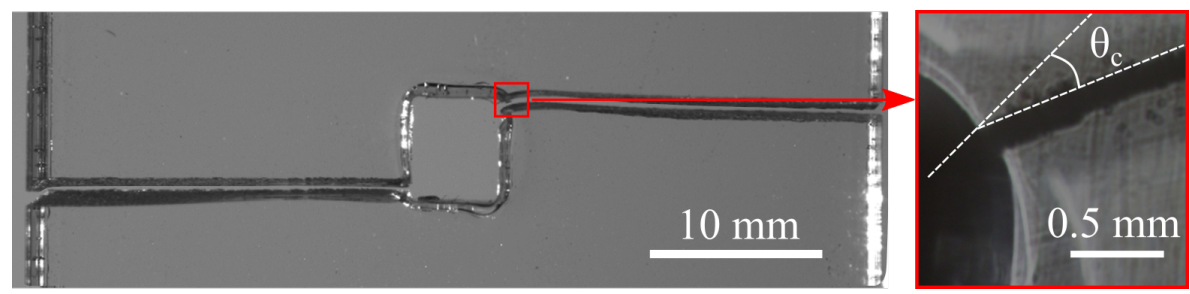

Fig. 7: Optical and microscope observation of a specimen with $9.39 \mathrm{~mm}$ square hole highlighting the crack deflection angle with respect to the $\mathrm{V}$-notch bissector.

The scattering in the measured deflections may be due to the fact that one of the two corner cracks initiates slightly before the other one, thus changing the mode mixity at the second initiation corner [24]. Despite a large 
scattering in the measured angles, it can be observed that the smaller the hole, the larger the crack deflection with respect to the notch bisector. Indeed, no deflection with respect to the V-notch bisector would be expected in the case of a pure opening mode. Therefore, it can be concluded that the larger the hole size, the smaller the mode mixity $\psi$. A more detailed analysis will be carried out in Section 4.2 , where the MA approach will be implemented and the GSIFs will be evaluated numerically.

\section{Finite Fracture Mechanics implementation}

\subsection{The coupled criterion}

The $\mathrm{CC}$ states that two conditions must be simultaneously fulfilled in order to ensure the nucleation of a crack. The first condition results from a balance between the states prior to and after crack initiation of the potential energy $\left(W_{p}\right)$, the kinetic energy $\left(W_{k}\right)$ and the crack surface energy $\left(G_{c} l, G_{c}\right.$ being the material fracture toughness).

$$
\delta W_{p}+\delta W_{k}+G_{c} l=0
$$

For a quasi-static initial state, $\delta W_{k} \geq 0$, which results in a necessary condition for crack initiation:

$$
-\delta W_{p} \geq G_{c} l
$$

The second condition consists in ensuring that the stress normal to the crack plane $\sigma_{n n}$ overcomes the material strength along all the crack path (which is unknown a priori). In the classical case of a monotonically decreasing stress (for instance near a singular point such as a V-notch tip), this condition provides an upper bound for the initiation crack length, whereas the first condition (Eqn. (2)) provides a lower bound. In 2D, under the assumption of a straight initiation crack, the crack topology can be described by two parameters, namely its length $l$ and its deflection with respect to the V-notch bisector $\theta$ (Fig. 8a). Denoting the stress imposed to the specimen with $\sigma_{0}$, the stress and energy conditions thus write:

$$
\left\{\begin{array}{l}
G_{i n c}\left(l, \theta, \sigma_{0}\right)=-\frac{\delta W_{p}\left(l, \theta, \sigma_{0}\right)}{l} \geq G_{c} \\
\sigma_{n n}\left(l, \theta, \sigma_{0}\right) \geq \sigma_{c}
\end{array}\right.
$$

where $G_{i n c}$ is the incremental energy release rate. It can be noted that an average stress condition over the presupposed crack path $\Gamma$ can also be employed [4,5,21, which consists in replacing the stress criterion given 
in Eqn. (3) by the following condition:

$$
\frac{1}{l} \int_{\Gamma} \sigma_{n n}\left(l, \theta, \sigma_{0}\right) d l \geq \sigma_{c}
$$

For the sake of simplicity, the CC formulation is presented employing the stress criterion in the sequel, but the same reasoning holds true if its average form is adopted. The choice of employing either the stress criterion or its average form will be discussed in the following sections.

According to the CC, the crack initiates for the smallest loading according to which both energy and stress conditions are fulfilled. Therefore, the failure stress $\sigma_{f}$ can be determined by solving the following problem:

$$
\sigma_{f}=\min \left\{\sigma_{0}, \exists(l, \theta),\left(\frac{G_{i n c}\left(l, \theta, \sigma_{0}\right)}{G_{c}} \geq 1\right) \wedge\left(\frac{\sigma_{n n}\left(l, \theta, \sigma_{0}\right)}{\sigma_{c}} \geq 1\right)\right\}
$$

The critical crack initiation length $l_{c}$ and deflection $\theta_{c}$ thus verify:

$$
\min \left(\frac{G_{i n c}\left(l_{c}, \theta_{c}, \sigma_{f}\right)}{G_{c}}, \frac{\sigma_{n n}\left(l_{c}, \theta_{c}, \sigma_{f}\right)}{\sigma_{c}}\right)=1
$$

\subsection{Finite element implementation for circular hole}

A 2D plane strain FE model of circular hole specimens is set-up using Abaqus ${ }^{\mathrm{TM}}$ standard and Python scripts. The geometrical symmetry allows modeling only half the specimen, thus supposing a symmetric crack propagation . Symmetry condition with respect to (Oy) axis is imposed on the specimen middle edges and displacement of the specimen top face $\left(y=\frac{L}{2}\right)$ is prescribed. Fig. 8 p depicts a typical FE mesh of a circular hole specimen, consisting of 4-node linear elements and typically exhibiting around 20000 nodes and elements. Linear elastic isotropic material behavior is employed, with Young's modulus $E=2.7 \mathrm{GPa}$ and Poisson's ratio $\nu=0.39$. Under

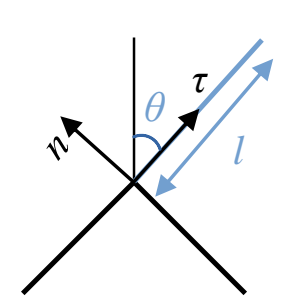

(a)

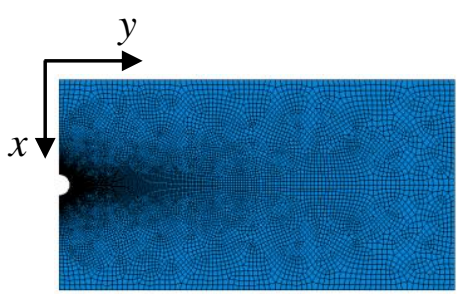

(b)

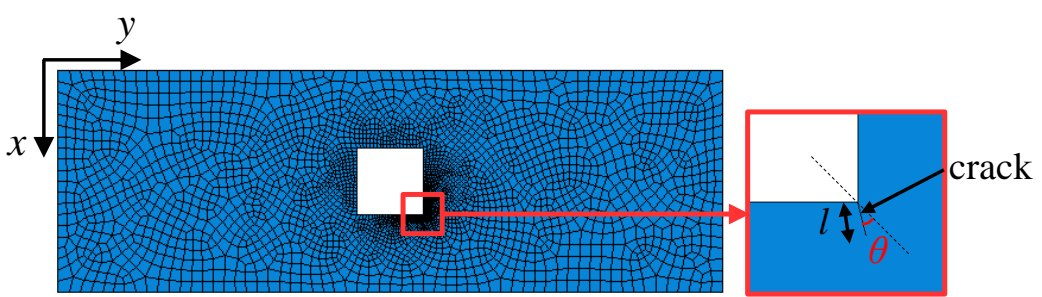

(c) (d)

Fig. 8: (a) V-notch crack representation. FE mesh for (b) circular and (c) square hole specimens; (d) focus on the square hole $\mathrm{V}$-notch (the mesh has been removed, for the sake of clarity). 
linear elasticity and small deformations assumption, the stress normal to the crack plane is proportional to the imposed stress whereas the incremental energy release rate is proportional to the square imposed stress. In formulae:

$$
\left\{\begin{array}{l}
G_{i n c}\left(l, \theta, \sigma_{0}\right)=A(l, \theta) \sigma_{0}^{2} \geq G_{c} \\
\sigma_{n n}\left(l, \theta, \sigma_{0}\right)=\xi(l, \theta) \sigma_{0} \geq \sigma_{c}
\end{array}\right.
$$

where $\xi$ and $A$ are functions that only depend on the structure geometrical features. The studied configuration leads to pure mode I crack initiation so that the crack orientation ((Oy) axis on Fig. 8) is known a priori. Moreover, the stress and the incremental energy release rate are respectively strictly increasing and decreasing functions of the crack length. Therefore Eqn. (6) reverts to the following equation:

$$
\frac{A\left(l_{c}\right)}{\xi\left(l_{c}\right)^{2}}=\frac{G_{c}}{\sigma_{c}^{2}},
$$

which can be solved employing Newton-Raphson algorithm to determine the initiation length $l_{c}$. The failure load can be determined using Eqn. (7):

$$
\sigma_{f}=\sqrt{\frac{G_{c}}{A\left(l_{c}\right)}}=\frac{\sigma_{c}}{\xi\left(l_{c}\right)}
$$

The FE implementation of the CC requires the function $A(l)$ and $\xi(l)$ to be computed in order to solve Eqn. (8). Only one calculation without crack is sufficient to compute $\xi$ along the crack path, whereas several calculations with varying crack lengths $l$ are required to obtain $A$. For circular hole specimens, this is done by progressively releasing the symmetry condition with respect to (Oy) axis along the crack path.

Note that an advantage of the CC is that the fracture parameters $G_{c}$ and $\sigma_{c}$ are implemented during the postprocessing phase. Indeed, once $A$ and $\xi$ are computed, the CC solution for given properties $\left(G_{c}, \sigma_{c}\right)$ only requires solving Eqn. (8) without any additional FE calculations. This is especially convenient for material parameter identification by a comparison with experimental measurements (which will be described in Section 4.1), since the solution for several material properties can be obtained through a quick post-processing.

3.3 Finite element implementation for square holes

A 2D plane strain FE model of square hole specimens is set-up using Abaqus ${ }^{\mathrm{TM}}$ (Fig. 8f-d). The same linear elastic isotropic material behavior as for circular hole case is employed. The main difference in the CC imple- 
mentation is that the crack deflection $\theta$ is not known a priori. However, for a fixed value of $\theta=\theta_{0}$, the same reasoning can be employed. The equation that must be solved to determine $l_{c}\left(\theta_{0}\right)$ is:

$$
\frac{A\left(l, \theta_{0}\right)}{\xi\left(l, \theta_{0}\right)^{2}}=\frac{G_{c}}{\sigma_{c}^{2}}
$$

It can be noted that, contrary to the circular hole specimens, the crack path does not lie on a symmetry axis, preventing the crack length to be varied by releasing symmetry conditions. In such a case, the crack length is varied by progressively doubling the nodes along the crack path in order to compute $A\left(l, \theta_{0}\right)$. The failure stress can thus be determined from Eqn. [7):

$$
\sigma_{f}\left(\theta_{0}\right)=\sqrt{\frac{G_{c}}{A\left(l_{c}\left(\theta_{0}\right), \theta_{0}\right)}}=\frac{\sigma_{c}}{\xi\left(l_{c}\left(\theta_{0}\right), \theta_{0}\right)}
$$

Finally, the failure crack deflection $\theta_{c}$ is obtained as the one minimizing the failure stress $\sigma_{f}(\theta)$. Fig. 9 depicts an example of failure stress as a function of the crack deflection, whose variation exhibits a minimum corresponding to the failure crack deflection and stress.

Compared to the circular hole case, solving the $\mathrm{CC}$ for square hole specimens requires more $\mathrm{FE}$ calculations because the crack is described by two parameters (the crack deflection and the crack length) instead of one (the crack length). However, this approach remains efficient even in this case. On the one hand, similarly to circular hole case, the material strength and fracture toughness are only implemented during the post-processing step.

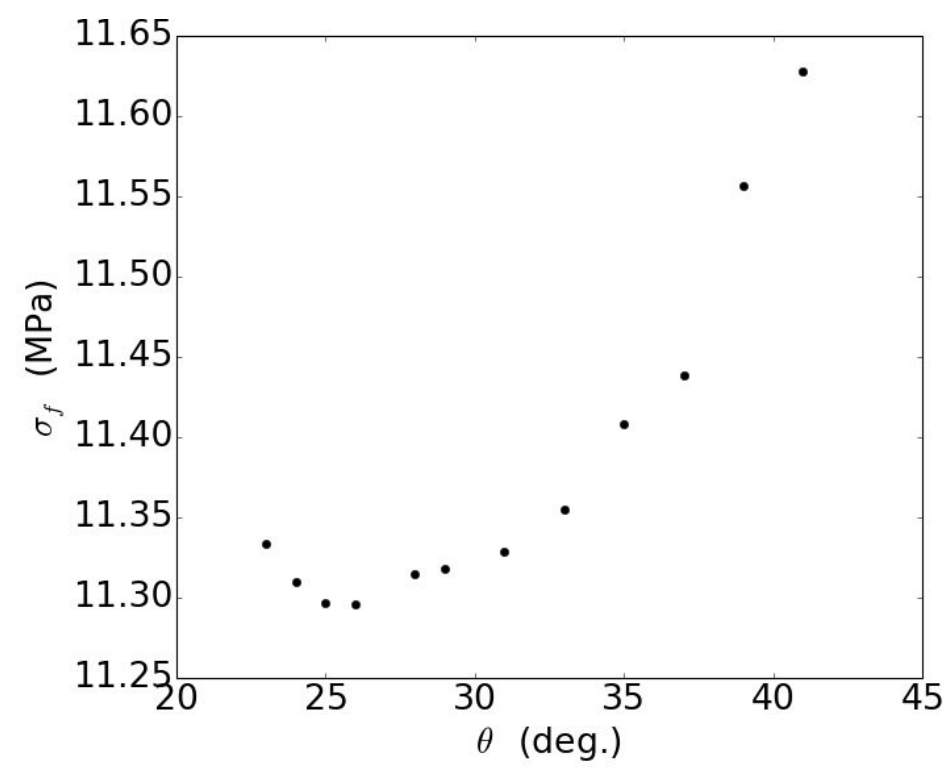

Fig. 9: Failure stress predicted by the $\mathrm{CC}$ as a function of the crack deflection angle. 
On the other hand, several calculations for different crack orientations can be executed simultaneously so that the effective calculation time does increase too much compared to the case for which the crack is described by only one parameter.

\section{Results and discussions}

\subsection{Parameter identification}

Instead of characterizing the material strength and fracture toughness separately through different fracture tests, we choose to determine these parameters based on the experimental tests on circular hole specimens with different hole diameters (Table 1). We take advantage of the fact that:

i) When the hole size tends towards zero, the specimen behaves as if it is plain, hence the failure stress tends towards the material strength. From a theoretical point of view, any hole radius induces a decrease in the failure stress. However, fixing an engineering tolerance of $5 \%$, it has been recently proved that for (mode I) tensile geometries the structure is:

- feature shape insensitive for sizes smaller $0.53 \mathrm{~mm}$ (according to the present material, i.e. extruded PMMA): in this case, the notch can be treated as a crack of the same size [25].

- feature shape insensitive for sizes smaller than approximately $0.02 \mathrm{~mm}$. In this case, the failure stress is not affected by the presence of a feature, whatever this is, and which of course can be neglected [25].

ii) When the hole size is big enough, crack initiation at the circular hole almost reverts to the configuration of a straight edge. In this case, failure is governed by the stress criterion and since the stress concentration at the hole corresponds to $3 \sigma_{0}$, the failure stress tends towards $\sigma_{f}=\frac{\sigma_{c}}{3}$ when the hole size increases (keeping small with respect to the other geometrical dimensions).

iii) For intermediate hole sizes, the specimen failure does not depend only on the material strength, but is also governed by the fracture toughness.

The proposed method for parameter identification relies on numerical modeling of the experimental test. The two unknown material parameters can be thus estimated in order to best reproduce the failure stress variation as a function of the circular hole size. A main advantage of the coupled criterion is its ability to reproduce the size effects observed experimentally, employing as input parameters only the material Young's modulus, Poisson's ratio, strength and fracture toughness. The two first parameters being already determined from experimental 
tests on specimens without hole, the only parameters that can be adjusted are the material strength and fracture toughness. The parameter identification is obtained by least square minimization of the difference between measured and predicted circular hole specimen failure forces. Employing the stress criterion yields $G_{c}=92 \mathrm{~J} / \mathrm{m}^{2}$ and $\sigma_{c}=42.9 \mathrm{MPa}$. On the other hand, if the average stress criterion is implemented, the obtained parameters are slightly different: $G_{c}=140 \mathrm{~J} / \mathrm{m}^{2}$ and $\sigma_{c}=45 \mathrm{MPa}$. The obtained order of magnitude lies within the typical parameter range for extruded PMMA. Fig. 10 depicts the failure stress variation as a function of the hole diameter obtained with these parameters. They will be used in next section for blind predictions of square hole specimen failure.

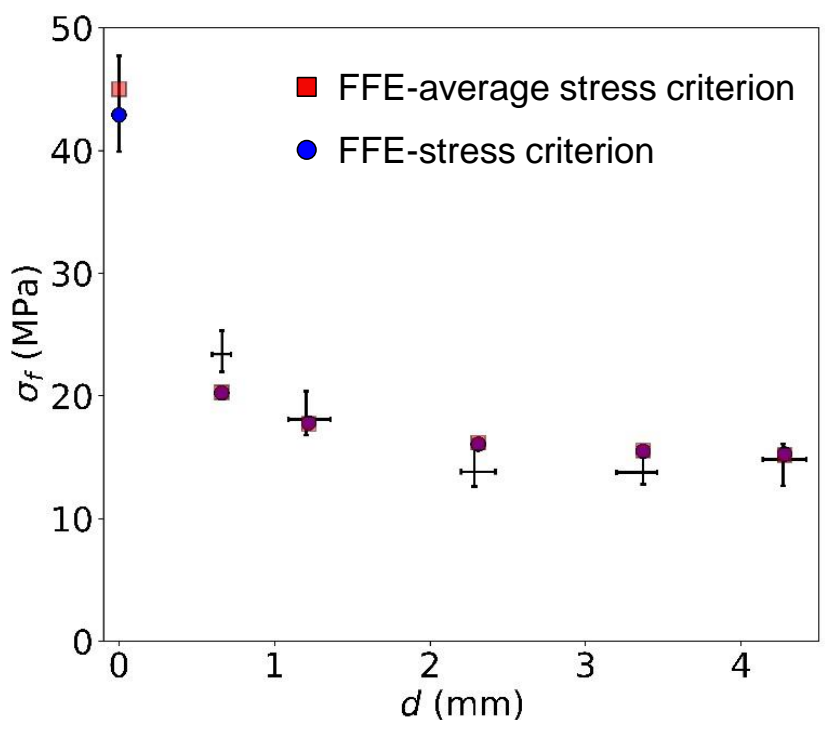

Fig. 10: Failure stress predicted by the CC employing the stress criterion (blue dots, $\sigma_{c}=42.9 \mathrm{MPa}, G_{c}=92 \mathrm{~J} / \mathrm{m}^{2}$ ) or its average version (red squares, $\sigma_{c}=45 \mathrm{MPa}, G_{c}=140 \mathrm{~J} / \mathrm{m}^{2}$ ) as a function of the circular hole diameter.

\subsection{Blind predictions on square holes}

The $\mathrm{CC}$ is now applied to the experimental data related to square hole specimens (Section 2.2). It must be emphasized that the numerical results represent blind predictions using the methods described in Section 3 and the parameters identified in Section 4.1 i.e. $\left(G_{c}=92 \mathrm{~J} / \mathrm{m}^{2}, \sigma_{c}=42.9 \mathrm{MPa}\right)$ using the stress criterion and $\left(G_{c}=140 \mathrm{~J} / \mathrm{m}^{2}, \sigma_{c}=45 \mathrm{MPa}\right)$ using its average version. If the same parameters are implemented, it is consolidated that the stress criterion involves a smaller critical length than that related to the average stress criterion, thus providing larger failure loads and larger propagation angles in case of mixed mode loading conditions [21]. However, in the present study different parameters are implemented for the two criteria: the above considerations 


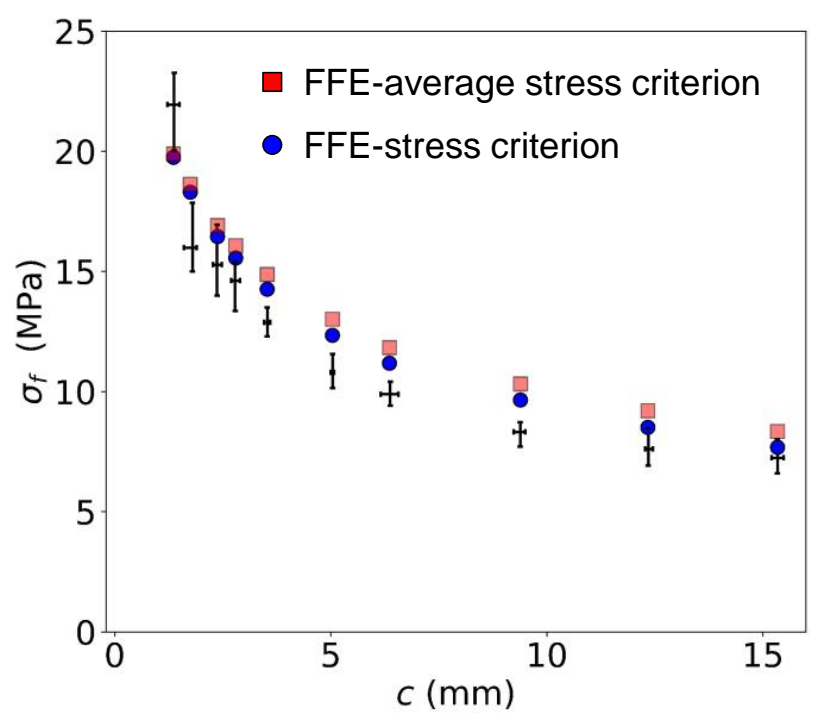

(a)

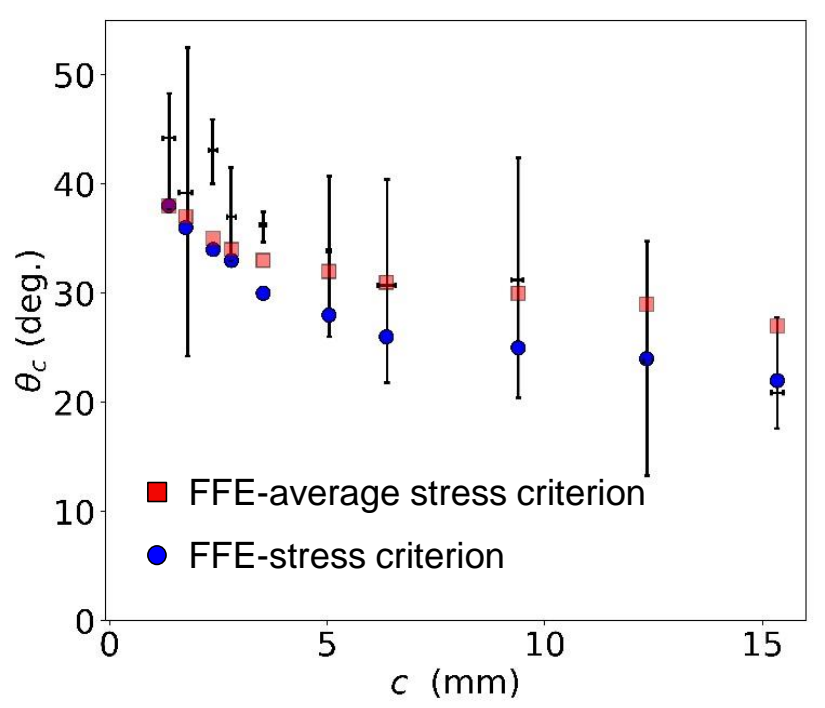

(b)

Fig. 11: (a) Failure stress and (b) crack deflection as a function of the square hole size: experiments (error bars) and FFE predictions by the stress criterion (blue dots) or its average version (red squares).

are no longer valid, provided that the critical distance related to the stress criterion always reveals smaller. The predicted failure stress and crack deflection variations as a function of the hole side are shown in Fig 11 Using either the stress criterion or its average form, the size effect is well reproduced, i.e. the failure stress decreases increasing the square hole size. Indeed, both criteria slightly overestimate $\sigma_{f}$ (the percentage deviation being generally lower than $+16 \%$, with a maximum of $+20.4 \%$ for $c=9.39 \mathrm{~mm}$ ), except for the smallest hole (the error being nearly $-9 \%$ ). On the other hand, the crack deflection decreases for increasing square hole sizes. In this case, the average stress criterion allows obtaining a slight better agreement.

In Figure 12 we have considered the average stress criterion, and compared its predictions by implementing the FFE (as in Fig. 11] and the MA approach (see, for instance, [10]). Note that results by both approaches are very close ( $c f$. Fig 12 : as concerns the failure load, the maximum percentage deviation always keeps below $2 \%$, whereas it is generally below $3 \%$ for the crack deflection angle. Regarding the MA approach and following, for instance, the analysis performed in [5, 14], the mode mixity $\psi$ results from the values of the GSIFs $K_{I}^{*}$ and $K_{I I}^{*}$ through to the relationship:

$$
\psi=\arctan \frac{K_{I I}^{*}}{K_{I}^{*}} l_{c h}^{\lambda_{I I}-\lambda_{I}}
$$

where $l_{c h}=G_{c} E /\left(\sigma_{c}^{2}\left(1-\nu^{2}\right)\right) \approx 0.221 m m$, and $\lambda_{I}=0.5445, \lambda_{I I}=0.9085$ are the Williams' eigenvalues.

The GSIFs $K_{I}^{*}$ and $K_{I I}^{*}$ were estimated numerically and their values are reported in Table 3 . Consequently, Eq. (12) tells us that the mode mixity $\psi$ varies between nearly $16^{\circ}$ (for the largest hole) to nearly $31^{\circ}$ (for 


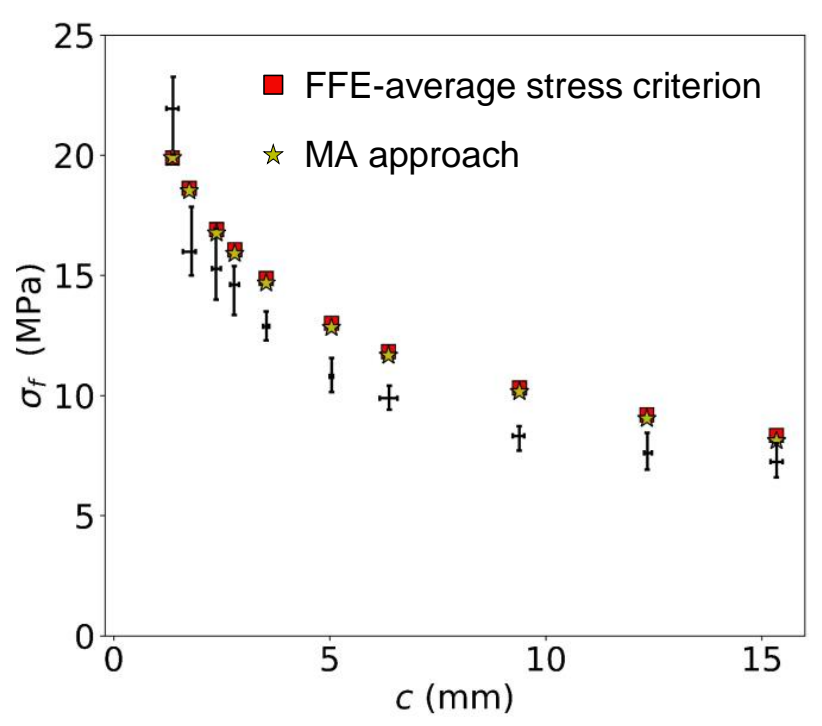

(a)

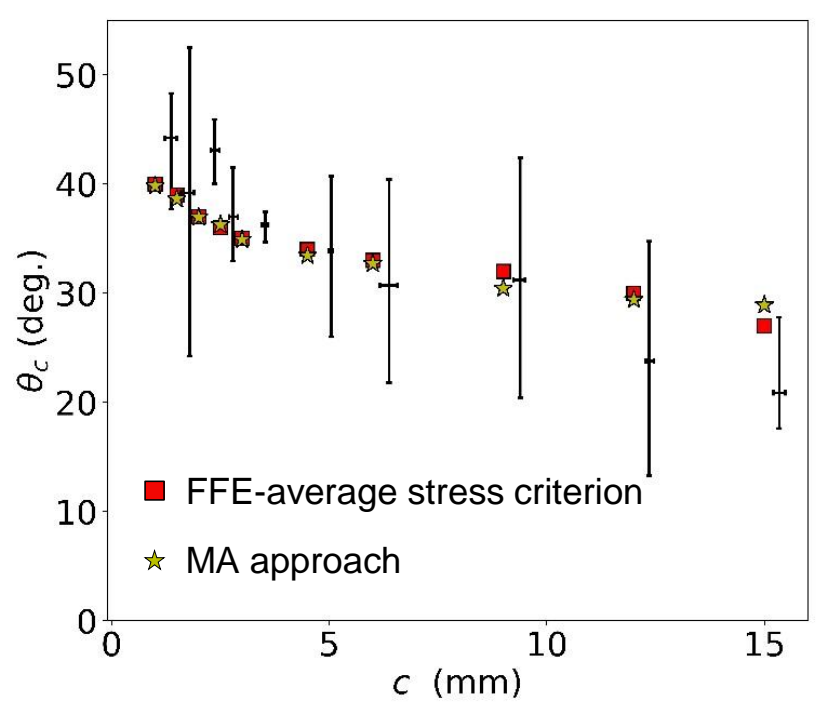

(b)

Fig. 12: (a) Failure stress and (b) crack deflection as a function of the square hole size: experiments (error bars) and numerical predictions employing the MA ([5], yellow stars) or the FFE approach with the average stress criterion (red squares).

the smallest hole): as the size decreases, the influence of mode II increases. By considering smaller holes, the propagation angle would approach $45^{\circ}$. Slightly predominant mode II fracture conditions can be achieved for very small sizes (smaller than approximately $0.7 \mathrm{~mm}$ ). However, note that if the hole disappears or can be neglected, the crack angle is effectively $45^{\circ}$ (with respect to the present axis system) and the structure reverts to a tensile plain specimen, thus under pure mode I loading conditions. In Table 3 the critical values of the mode I GSIF $K_{I f}^{*}$ are also reported, for the sake of completeness.

\begin{tabular}{|c|c|c|c|c|c|c|c|c|c|c|}
\hline Hole side $c(\mathrm{~mm})$ & 1.36 & 1.75 & 2.38 & 2.80 & 3.53 & 5.04 & 6.36 & 9.39 & 12.34 & 15.34 \\
\hline$K_{I}^{*}\left(\mathrm{MPa} \mathrm{mm}^{1-\lambda_{I}}\right)$ & 0.8015 & 0.8962 & 1.032 & 1.111 & 1.236 & 1.460 & 1.631 & 1.943 & 2.211 & 2.453 \\
\hline$K_{I I}^{*}(\mathrm{MPa} 1$ & 0.8459 & 0.8577 & .8826 & 0.8932 & 0.9145 & 0.9689 & 1.019 & 1.039 & 1.111 & .191 \\
\hline$\psi(\operatorname{deg})$ & 31.4 & 28.9 & 26.3 & 24.9 & 23.1 & 21 & 19 & 17 & 16 & 15.7 \\
\hline$K_{I f}^{*}\left(\mathrm{MPa} \mathrm{mm}{ }^{1-\lambda_{I}}\right)$ & 15.95 & 16.59 & 17.31 & 17.67 & 18.14 & 18.72 & 19.02 & 19.73 & 19.99 & 20.11 \\
\hline
\end{tabular}

Table 3: Square hole samples under uniaxial loading: GSIF values and related mode mixity. The critical values of the mode I GSIFs are also reported.

\section{Conclusions}

Uniaxial tensile tests were carried out on PMMA specimens containing a square hole. This kind of experiments allows to get mixed mode failure: the mode mixity depends on the hole size, the amount of opening mode I increasing with increasing hole sizes. Experimental measurements on the crack deflection with respect to the 
V-notch bisector exhibited a large scatter, nevertheless highlighting the trend described above.

Once evaluated the material Young's modulus and Poisson's ratio, the material strength and fracture toughness were determined by an indirect confrontation of the CC predictions on experimental failure stresses related to circular holes with different diameters. This approach proves to be computationally really efficient, since it only requires varying the material properties during the post-processing stage.

Blind predictions on the failure stress and the crack deflection variation as a function of the square hole side obtained by applying the CC through a FFE approach, both in the form proposed by Leguillon [16] and in that proposed by Carpinteri et al. 4, were in good agreement with experimental data. Finally, it was shown that FFE results are very close to those provided by the MA approach, which thus represents an efficient tool for the geometry under investigation.

\section{References}

1. Ayatollahi, M.R., Aliha, M.R.M. (2009) Analysis of a new specimen for mixed mode fracture tests on brittle materials. Engng. Fract. Mech. 76, 1563-1573.

2. Ayatollahi, M.R., Torabi, A.R., Bahrami, B. (2016) On the necessity of using critical distance model in mixed mode brittle fracture prediction of V-notched Brazilian disk specimens under negative mode I conditions. Theor. App. Fract. Mech. 84, $38-48$.

3. Carrère, N., Martin, E., Leguillon, D (2015) Comparison between models based on a coupled criterion for the prediction of the failure of adhesively bonded joints. Engng. Fract. Mech. 138, 185-201.

4. A. Carpinteri, P. Cornetti, N. Pugno, A. Sapora and D. Taylor (2008) A finite fracture mechanics approach to structures with sharp V-notches. Engng. Fract. Mech. 75, 1736-1752.

5. Cornetti, P., Sapora, A., Carpinteri, A. (2013) Mode mixity and size effect in V-notched structures. Int. J. Sol. Struct. 50(10), $1562-1582$.

6. Doitrand, A., Fagiano, C., Carrère, N., Chiaruttini, V., Hirsekorn, M. (2017) Damage onset modeling in woven composites based on a coupled stress and energy criterion. Engng. Fract. Mech. 169, 189-200.

7. Doitrand, A., Leguillon, D. (2018) 3D application of the coupled criterion to crack initiation prediction in epoxy/aluminum bimaterial specimens under four point bending. Int. J. Sol. Struct. 143, 175-182.

8. Doitrand, A., Leguillon, D.(2018) Numerical modeling of the nucleation of facets ahead of a primary crack under mode I+III loading. Int. J. Fract. 213, 37-50.

9. Doitrand, A., Estevez, R., Leguillon, D (2019) Experimental characterization and numerical modeling of crack initiation in rhombus hole PMMA specimens under compression. Eur. J. Mech. A/Sol. 76, 290-299.

10. Doitrand, A., Martin, E., Leguillon, D. (2020) Numerical implementation of the coupled criterion: Matched asymptotic and full finite element approaches. Fin. Elem. Anal. Des. 168, 103344. 
11. Felger, J., Rosendahl, P.L. Leguillon, D. Becker, W. (2019) Predicting crack patterns at bi-material junctions: A coupled stress and energy approach. Int. J. Sol. Struct. 164, 191-201.

12. Gómez, F.J., Elices, M., Berto, F., Lazzarin, P. (2009) Fracture of V-notched specimens under mixed mode (I + II) loading in brittle materials. Int. J. Fract. 159, 121-135.

13. Hashin, Z. (1996) Finite thermoelastic fracture criterion with application to laminate cracking analysis. J. Mech. Phys. Sol. $44(7), 1129-1145$.

14. Hills, D.A., Dini, D. (2011) Characteristics of the process zone at sharp notch roots. Int. J. Sol. Struct. 48, $2177-2183$.

15. D. Leguillon, E. Sanchez-Palencia (1987) Computation of singular solutions in elliptic problems and elasticity, John Wiley, New York.

16. D., Leguillon (2002) Strength or toughness ? A criterion for crack onset at a notch, Eur. J. Mech. Sol. 21, 61-72.

17. Martin, E., Poitou, B., Leguillon, D., Gatt, J.M. (2008) Competition between deflection and penetration at an interface in the vicinity of a main crack. Int. J. Fract. 151(2), 247-268.

18. Nairn, J.A. (2000) Exact and variational theorems for fracture mechanics of composites with residual stresses, traction-loaded cracks and imperfect interfaces. Int. J. Fract. 105, 243-271.

19. Priel E, Bussiba A, Gilad I, Yosibash Z (2007) Mixed mode failure criteria for brittle elastic V-notched structure. Int. J. Fract. $144,247-265$.

20. E. Priel, Z. Yosibash, D. Leguillon (2008) Failure initiation at a blunt V-notch tip under mixed mode loading, Int. J. Fract. $149,143-173$.

21. Sapora, A., Cornetti, P., Carpinteri, A. (2013) A Finite Fracture Mechanics approach to V-notched elements subjected to mixed-mode loading. Engng. Fract. Mech. 97, 216-226.

22. Seweryn, A., Lukaszewicz, A. (2002) Verification of brittle fracture criteria for elements with V-shaped notches. Engng. Fract. Mech. 69, 1487- 1510.

23. Z. Yosibash, E. Priel, D. Leguillon (2006) A failure criterion for brittle elastic materials under mixed-mode loading, Int. J. Fract. 141, 291-312.

24. Doitrand, A., Leguillon, D., Estevez, R. (2020) Experimental determination of generalized stress intensity factors from full-field measurements. Engng. Fract. Mech. 206, 106980.

25. A. Sapora, P. Cornetti, A. Campagnolo, G. Meneghetti (2020) Fatigue limit: Crack and notch sensitivity by Finite Fracture Mechanics. Theor. App. Fract. Mech. 105, 102407.

26. Saad-Gouider, N., Estevez, R., Olagnon, C., Seguela, R (2006) Calibration of a viscoplastic cohesive zone for crazing in PMMA. Engng. Fract. Mech. 73(16), 2503-2522. 